\title{
Bounds on convex bodies in pairwise intersecting Minkowski arrangement of order $\mu$
}

\author{
Viktória Földvári®i
}

\begin{abstract}
The $\mu$-kernel of an $o$-symmetric convex body is obtained by shrinking the body about its center by a factor of $\mu$. As a generalization of pairwise intersecting Minkowski arrangement of $o$-symmetric convex bodies, we can define the pairwise intersecting Minkowski arrangement of order $\mu$. Here, the homothetic copies of an $o$-symmetric convex body are so that none of their interiors intersect the $\mu$-kernel of any other. We give general upper and lower bounds on the cardinality of such arrangements, and study two special cases: For $d$-dimensional translates in classical pairwise intersecting Minkowski arrangement we prove that the sharp upper bound is $3^{d}$. The case $\mu=1$ is the Bezdek-Pach Conjecture, which asserts that the maximum number of pairwise touching positive homothetic copies of a convex body in $\mathbb{R}^{d}$ is $2^{d}$. We verify the conjecture on the plane, that is, when $d=2$. Indeed, we show that the number in question is four for any planar convex body.
\end{abstract}

Mathematics Subject Classification. 52C15, 51N20, 52A37.

Keywords. Minkowski arrangement, Bezdek-Pach conjecture, homothets, translates, packing.

\section{Introduction}

A positive homothetic copy of a convex body (i.e. a compact convex set with non-empty interior) $K$ in Euclidean $d$-space $\mathbb{R}^{d}$ is a set of the form $\lambda K+t$ where $\lambda>0$ and $t \in \mathbb{R}^{d}$. Two sets in $\mathbb{R}^{d}$ are said to touch each other if they intersect but their interiors are disjoint.

The following notion was introduced by Fejes Tóth [10]: Pairwise intersecting homothets of a centrally symmetric convex body in the $d$-dimensional Euclidean space form a Minkowski arrangement if none of them contains the 
center of any other in its interior. In this paper, we only consider Minkowski arrangements that are pairwise intersecting.

Polyanskii [19] recently proved that such a family of convex bodies has at most $3^{d+1}$ members. This result was improved by Naszódi and Swanepoel [16] showing an upper bound of $2 \cdot 3^{d}$. It is natural to conjecture that the maximum number of elements is $3^{d}$.

We prove the following upper bound on the cardinality of a family containing translates of a centrally symmetric convex body in pairwise intersecting Minkowski arrangement in Sect. 2:

Theorem 1.1. In $\mathbb{R}^{d}$, a pairwise intersecting Minkowski arrangement consisting of translates of a centrally symmetric convex body $K$ contains at most $3^{d}$ elements. This bound is sharp, equality holds if and only if $K$ is a d-dimensional parallelotope.

We show a construction for arbitrary centrally symmetric convex body that gives a linear lower bound on the cardinality of maximal pairwise intersecting Minkowski arrangements of translates. Although from Theorem 3 of [1] we can deduce the existence of an exponential lower bound, we now give a simple and deterministic construction.

Proposition 1.2. For a centrally symmetric convex body $K$ in $\mathbb{R}^{d}(d \geq 2)$, a maximum cardinality set consisting of translates of $K$ in pairwise intersecting Minkowski arrangement has at least $2 d+3$ elements.

We introduce some generalizations of the problem based on an idea of Fejes Tóth [11], examined by Böröczky and Szabó [6,8]: For $0 \leq \mu \leq 1$ they defined the $\mu$-kernel of an $o$-symmetric convex body $K$ as $\mu K$.

Using this notion, for homothets of an o-symmetric convex body we can consider a pairwise intersecting Minkowski arrangement of order $\mu$, where the homothets are pairwise intersecting but none of their interiors intersect the center of any other.

We prove an upper bound on the cardinality of such an arrangement, then, for centrally symmetric convex bodies we verify the existence of an exponential lower bound.

Theorem 1.3. In $\mathbb{R}^{d}$, a pairwise intersecting Minkowski arrangement of order $\mu$ consisting of translates of a centrally symmetric convex body $K$ contains at most $\left(1+\frac{2}{1+\mu}\right)^{d}$ elements.

Proposition 1.4. Let $M_{\mu}(K)$ denote the maximum number of translates of a $d$ dimensional, o-symmetric convex body $K$ in pairwise intersecting $\mu$-Minkowski arrangement. For $\mu<\sqrt{2}-1$, there exists a lower bound $M_{\mu}(K) \geq e^{c d}$ for some universal constant $c$. 
In 1962, Danzer and Grünbaum [9] proved that the maximum cardinality of a family of pairwise touching translates of a convex body $K$ in $\mathbb{R}^{d}$ is $2^{d}$, which bound is attained if and only if $K$ is an affine image of a cube. Petty [18] showed that every convex body in the plane (or in 3-space) has three (four) pairwise touching translates. As an extension of this problem, Bezdek and Pach [5] conjectured in 1988 that the maximum number of pairwise touching positive homothetic copies of a convex body in $\mathbb{R}^{d}$ is $2^{d}$. They showed that any such family of homothetic copies has at most $3^{d}$ elements, and if $K$ is a $d$-dimensional Euclidean ball, then the maximum is equal to $d+2$. Naszódi [15] improved the first estimate by proving the upper bound $2^{d+1}$. In [14], Lángi and Naszódi proved (using a result [4] of Bezdek and Brass about one-sided Hadwiger numbers) the upper bound $3 \cdot 2^{d-1}$ in the case when $K$ is centrally symmetric.

In Sect. 3, we show that the conjecture holds on the plane, moreover, every planar convex body has four pairwise touching homothets.

Theorem 1.5. For any convex body $K$ in $\mathbb{R}^{2}$, the maximum number of pairwise touching positive homothetic copies of $K$ is four.

The generalized notion of Minkowski arrangement provides a connection between the original problem of pairwise intersecting Minkowski arrangements and the Bezdek-Pach Conjecture [5]. In both problems we consider pairwise intersecting Minkowski arrangements of order $\mu$, in the first case $\mu=0$, while in the latter case $\mu=1$.

For two points $a, b$ in $\mathbb{R}^{d}$, we denote the closed and the open line segment connecting them by $[a, b]$ and $(a, b)$, respectively. We use the standard notations conv, bd and int to denote the convex hull, the boundary and the interior of a set in $\mathbb{R}^{d}$, respectively.

In Sect. 2, we prove Theorems 1.1, 1.3, and Propositions 1.2 and 1.4. Sections 3 and 4 together give the proof of Theorem 1.5. Finally, in Sect. 5, we verify Proposition 5.1, a topological note that yields to an alternative version of the proof of Theorem 1.5.

\section{Bounds on pairwise intersecting Minkowski arrangements}

It is natural to conjecture that in $\mathbb{R}^{d}$, a pairwise intersecting Minkowski arrangement consisting of homothets of a centrally symmetric convex body contains at most $3^{d}$ elements. Here we prove this upper bound - and a generalization - for the case when all the homothets in the arrangement are translates of the given body. 


\subsection{Proofs of Theorems 1.1 and 1.3}

First, we verify Theorem 1.3, then Theorem 1.1 will follow as a corollary.

Any $o$-symmetric convex body $K$ can be considered as the unit ball of a normed space $\left(\mathbb{R}^{d},\|\cdot\|_{K}\right)$, where for any $x \in \mathbb{R}^{d},\|x\|_{K}=\inf \left\{\lambda \in \mathbb{R}^{+} \mid x \in \lambda K\right\}$. It is easy to see that having a pairwise intersecting Minkowski arrangement of order $\mu$ is equivalent to the following two conditions on the distances between centers: none of them can be farther than 2 , nor closer than $1+\mu$ to any other. After applying a homothety, this is equivalent to the problem when the distances are between 1 and $\frac{2}{1+\mu}$.

Lemma 2.1. Consider a centrally symmetric convex body $K$ in $\mathbb{R}^{d}$ and $v_{1}, v_{2}$, $\ldots, v_{n} \in \mathbb{R}^{d}$, so that $1 \leq\left\|v_{i}-v_{j}\right\|_{K} \leq \lambda$ for any $i \neq j$. Then $n \leq(\lambda+1)^{d}$.

Proof. By the assumption, for different indices the bodies $v_{i}+\frac{1}{2} K$ are pairwise non-overlapping. Let $Q=\operatorname{conv}\left[\bigcup_{i=1}^{n}\left(v_{i}+\frac{1}{2} K\right)\right]$. Since $\operatorname{diam}_{K}(Q) \leq \lambda+1$, using the isodiametric inequality for Minkowski spaces [12] we get that

$$
\frac{n}{2^{d}} \operatorname{Vol}(K) \leq \operatorname{Vol}(Q) \leq \operatorname{Vol}\left(\frac{\lambda+1}{2} K\right) .
$$

From this, $n \leq(\lambda+1)^{d}$ follows.

Applying this lemma for $\lambda=\frac{2}{1+\mu}$, we get that the number of points with pairwise distances between 1 and $\frac{2}{1+\mu}$ is at most $\left(1+\frac{2}{1+\mu}\right)^{d}$, which is equivalent to the statement of Theorem 1.3.

Theorem 1.1 is the special case of Theorem 1.3 with $\mu=0$, so the upper bound $3^{d}$ follows easily.

To reach this, (2.1) has to hold with two equalities. From the following lemma of Groemer [13] we can see that this happens if and only if $K$ is a $d$-dimensional parallelotope.

Lemma 2.2. Suppose that $K$ is a convex body in $\mathbb{R}^{d}$ such that for some $1<$ $t \in \mathbb{R}$ the body $t K$ can be decomposed into translates of $K$. Then $K$ is a $d$-dimensional parallelotope and $t$ is an integer. The partition is unique.

Remark 2.3. The bound in Theorem 1.3 gives the known result $2^{d}$ for the pairwise touching case, when $\mu=1$.

\subsection{Proof of Proposition 1.2}

First, we show a construction of seven bodies in $\mathbb{R}^{2}$, then the lower bound $2 d+3$ for the higher dimensional cases will follow recursively. In $\mathbb{R}^{2}$, consider 


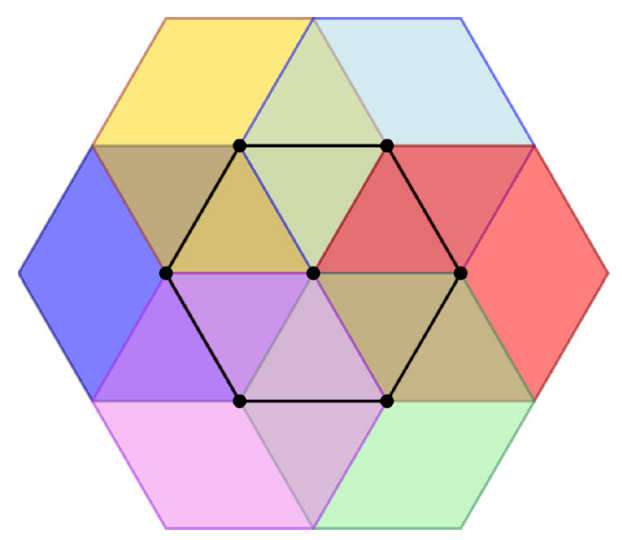

Figure 1 Seven translates of an affine-regular hexagon in Minkowski arrangement

an affine-regular hexagon inscribed in $K$ that is symmetric about the center of $K$ (see for example [17, Lemma 4.3]). There exist seven translates of this hexagon in Minkowski arrangement, shown in Fig. 1. Translate $K$ in a way that the center points are the same as the centers of the above hexagons. Now a center of any translate is either not contained in another body, or lies on its boundary. Furthermore, these translates share a common point, so they are pairwise intersecting. This means that the construction gives a Minkowski arrangement.

For a centrally symmetric convex body $K$ in $\mathbb{R}^{d}$, denote by $M(K)$ the maximal number of translates in a pairwise intersecting Minkowski arrangement. It is easy to see, that for any $K$ in $\mathbb{R}^{1}, M(K)=3$, and we showed that for $K$ in $\mathbb{R}^{2}, M(K) \geq 7$.

Let $e_{1}, \ldots, e_{d}$ be an Auerbach basis $\left[21\right.$, Chapter3] of the space $\left(\mathbb{R}^{d},\|\cdot\|_{K}\right)$. In dimension $d \geq 3$, using the above planar construction, we can take seven translates of $K$ in a Minkowski arrangement such that their centers lie in the plane of the first two basis vectors $e_{1}$ and $e_{2}$ : The plane spanned by $e_{1}$ and $e_{2}$ intersects a translate of $K$ whose center is in this plane in a centrally symmetric convex body $\widetilde{K}$. According to the above construction, we take seven translates of $\widetilde{K}$ as in Fig. 1 such that the center of the middle body is $o$. Now these translates of $\widetilde{K}$ are in a planar Minkowski arrangement. Then consider seven translates of $K$ with the same center points as the centers of the seven planar bodies. Since each of their centers lie in the same plane where they are either not contained in another body, or lie on its boundary, these translates of $K$ are also in a Minkowski arrangement. Along each direction $e_{3}, \ldots, e_{d}$ we can add two further translates of $K$ to this arrangement so that they contain $o$ on their boundary (for each $i=3, \ldots, d$, one needs to take the translates of the middle body by $\pm e_{i}$, this only intersects the plane spanned by other two basis vectors on its boundary). 
Now we verify Proposition 1.4. Note that Theorem 3. of [1] by Naszódi, Pach and Swanepoel implies the existence of an exponential lower bound for translates in pairwise intersecting Minkowski arrangement. Their idea was based on a result of Arias-de-Reyna, Ball, and Villa [2]. Here we give a similar argument for $\mu$-Minkowski arrangements.

Proof of Proposition 1.4. The statement follows from a result of Bourgain [7]. He showed that on the unit sphere of any normed space, there is an exponentially large number of points so that the distance of any two is more than $\sqrt{2}-\varepsilon$. Consider the $o$-symmetric convex body $K$ as the unit ball of the normed space $\left(\mathbb{R}^{d},\|\cdot\|_{K}\right)$. Choosing $\mu<\sqrt{2}-1$, we get exponentially many points on the sphere so that their pairwise distances are between $1+\mu$ and 2 . Considering these points as centers, we verify the statement.

\section{Proof of the upper bound in Theorem 1.5}

Let $\mathcal{K}=\left\{K_{1}, K_{2}, \ldots, K_{n}\right\}$ be a family of pairwise touching positive homothetic copies of a planar convex body $K$.

If there is a point that belongs to four of the homothets, then we can enlarge (or shrink) each of the four bodies from that point as a center, to obtain four touching translates of $K$. By the result of Danzer and Grünbaum [9], this implies that $K$ is a parallelogram. It is easy to see that in this case, the family does not have a fifth member. Thus, from this point on, we will assume that no point belongs to four of the homothets.

If there is a point that belongs to three of the homothets and $\mathcal{K}$ has at least four members, then we will show that this point also belongs to a fourth body.

Proposition 3.1. Let $K_{1}, K_{2}, K_{3}, K_{4}$ be pairwise touching positive homothets of the convex body $K$ in $\mathbb{R}^{2}$. If $K_{1} \cap K_{2} \cap K_{3} \neq \emptyset$, then $K_{1} \cap K_{2} \cap K_{3} \cap K_{4} \neq \emptyset$.

Proof. We can assume that $K$ contains the origin in its interior, and that $K_{i}=\lambda_{i} K+x_{i}$ for some $\lambda_{i} \in \mathbb{R}$ and $x_{i} \in \mathbb{R}^{2}$. Let $p \in K_{1} \cap K_{2} \cap K_{3}$, and $C_{i}$ be the smallest angular region with vertex $p$ containing $K_{i}$.

We show that int $C_{i} \cap \operatorname{int} C_{j}=\emptyset$ for any $i \neq j, i, j \in\{1,2,3\}$.

Suppose that for a pair $i \neq j$ there exists $c \in\left(\operatorname{int} C_{i} \cap \operatorname{int} C_{j}\right)$. Then the line $p c$ intersects the interior of both $K_{i}$ and $K_{j}$ because $C_{i}$ and $C_{j}$ are the smallest angular regions containing $K_{i}$ and $K_{j}$ respectively. Hence due to the convexity of the bodies, $K_{i}$ overlaps $K_{j}$, which is a contradiction.

Suppose that $K_{1} \cap K_{2} \cap K_{3} \cap K_{4}=\emptyset$. Then $p \notin K_{4}$, thus there exists a supporting line $\ell$ of $K_{4}$ that does not go through $p$ and separates $K_{4}$ from $p$. $K_{4}$ touches $K_{1}, K_{2}$ and $K_{3}$, hence each of these three bodies has a point in both of the closed half-planes bounded by $\ell$. From this it follows that $\ell$ intersects the angular regions $C_{1}, C_{2}$ and $C_{3}$. For every $i \in\{1,2,3\}, \ell \cap C_{i}$ is a connected 


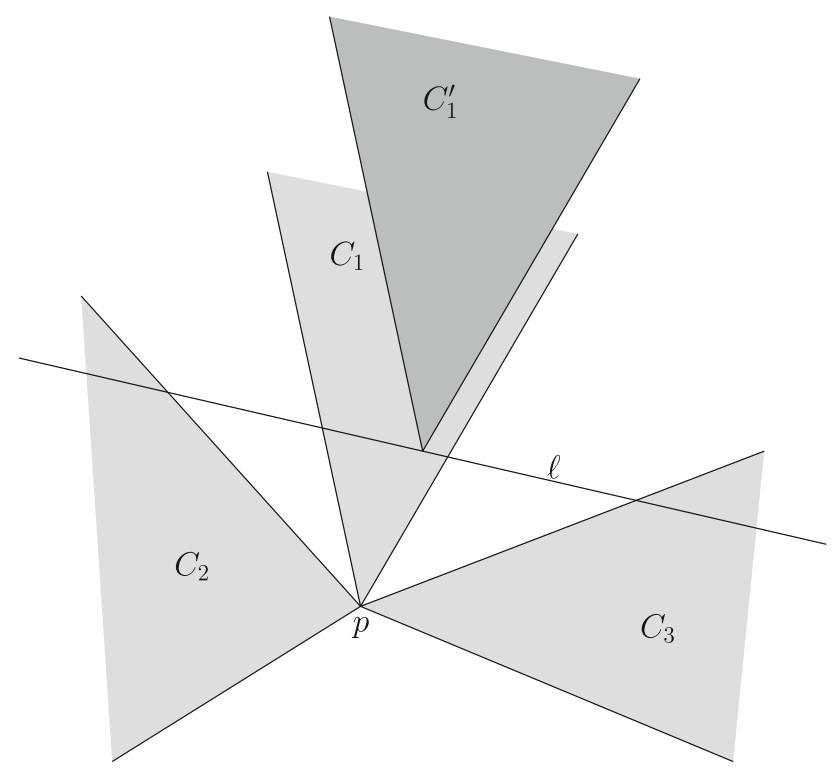

Figure 2 The smallest angular regions containing the bodies

subset of $\ell$, thus there is a middle one of them. Without loss of generality we can assume that this one is $K_{1}$. Let $v_{1}=p-x_{1}$. The image of $p$ by the homothety that maps $K_{1}$ to $K_{4}$ is the point $x_{4}+\frac{\lambda_{4}}{\lambda_{1}} \cdot v_{1} \in \ell$. The same homothety maps $C_{1}$ to the angular region $C_{1}^{\prime}:=C_{1}+\left(x_{4}-x_{1}+v_{1} \cdot\left(\frac{\lambda_{4}}{\lambda_{1}}-1\right)\right)$, see (Fig. 2). As $K_{1} \subset C_{1}$ and the bodies are positive homothets, $K_{4} \subset C_{1}^{\prime}$ follows. At least one pair of the bounding lines of $C_{1}$ and $C_{1}^{\prime}$ are different, thus due to the fact that int $C_{i} \cap \operatorname{int} C_{j}=\emptyset$ for any $i \neq j, i, j \in\{1,2,3\} C_{1}^{\prime}$ is disjoint from at least one of the angular regions $C_{2}$ and $C_{3}$. But in this case $K_{4}$ cannot touch the body lying in this angular region, which is a contradiction.

Thus, it is enough to consider the case when no point belongs to three of the homothets.

Proposition 3.2. Let $K_{1}, K_{2}, \ldots, K_{n}$ be pairwise touching convex bodies in $\mathbb{R}^{2}$, such that no three share a common point. Then $n \leq 4$.

Proof. For each $i \in\{1, \ldots, n\}$, choose an interior point $p_{i} \in K_{i}$. The bodies are pairwise touching, so we can draw a curve between any two of the chosen points $p_{i}, p_{j}$ so that it lies in $K_{i} \cup K_{j}$. Since no three of the bodies share a common point, these curves intersect only in the interior of the bodies. It is easy to see that we can eliminate these intersections with a perturbation. This way we draw the complete graph of $n$ vertices on the plane, from which $n \leq 4$ follows immediately. 


\section{Proof of the lower bound in Theorem 1.5}

In this section, we show that for any planar convex body $K$, there are four pairwise touching homothets of $K$.

Consider two distinct parallel support lines of $K$ that each touch $K$ at one point: $x_{1}$ and $x_{2}$. The existence of such a pair of lines follows from Theorem 2.2.9. of [20], but may also be proved as an exercise.

Let $K_{1}=K$ and $K_{2}=K+x_{2}-x_{1}$. Let $f$ be the line through the single point of contact, $x_{2}$, of $K_{1}$ and $K_{2}$ parallel to $x_{2}-x_{1}$. On both sides of $f$, there is a translate of $K$ that touches both $K_{1}$ and $K_{2}$. Indeed, if we push $K$ around $K_{1}$ so that it always touches $K_{1}$ then, by continuity, such two positions will be found.

If on both sides we can find such translates of $K$ that also contain $x_{2}$ then $x_{2}$ is a common point of four translates of $K$ and we are done. Thus we assume that at least one of these translates does not contain $x_{2}$. We call this translate $K_{3}$.

Now, $K_{1}, K_{2}, K_{3}$ are pairwise touching translates of $K$ that do not share a common point. It follows that they surround a bounded region $R$ with nonempty interior. Consider the largest homothet $K_{4}$ of $K$ contained in $R$. To finish the proof, we claim that $K_{4}$ touches $K_{1}, K_{2}$ and $K_{3}$. Indeed, assume $K_{4}$ touches only two of them, say $K_{1}$ and $K_{2}$. Consider a line that separates $K_{4}$ and $K_{1}$, and another line that separates $K_{4}$ and $K_{2}$. Let $u_{1}$ and $u_{2}$ be the unit normal vectors of these two lines respectively, pointing towards $K_{4}$. Clearly, if $u_{1}$ is not parallel to $u_{2}$, then $K_{4}$ can be moved a little inside $R$ (in the direction of $u_{1}+u_{2}$ ) so that it does not touch either $K_{1}, K_{2}$ or $K_{3}$. Then, we may enlarge $K_{4}$ slightly within $R$ contradicting the maximality of $K_{4}$. Thus $u_{1}$ is parallel to $u_{2}$. However, in this case, $K_{1}$ and $K_{2}$ are strictly separated, which is a contradiction, finishing the proof of the lower bound in Theorem 1.5.

\section{A topological note}

In this section we present Proposition 5.1, a topological observation, which may be used in place of Proposition 3.2 to prove the upper bound in Theorem 1.5.

An arc in the plane is the image of an injective continuous map of the $[0,1]$ interval into the plane. A Jordan curve in the plane is the image of an injective continuous map of the circle into the plane. We will call the closed bounded region bounded by a Jordan curve a Jordan region.

Let $K_{1}, K_{2}, K_{3}$ be three pairwise touching Jordan regions whose pairwise intersections are non-empty arcs (which may be degenerate, that is, a single point). Using the Jordan curve theorem, it is easy to show that if $K_{1} \cap K_{2} \cap K_{3}=\emptyset$, 
then the complement of $K_{1} \cup K_{2} \cup K_{3}$ in the plane has two connected components, one bounded and one unbounded. We call the closure of the bounded component the internal region surrounded by $K_{1}, K_{2}, K_{3}$, and the closure of the unbounded component the external region. (The idea of this statement is the following: If two Jordan curves intersect each other, then we can choose a meeting and a leaving point of each arc of their intersection. Cut each Jordan curve to arcs at its meeting and leaving points. Then, choosing some appropriate arcs of the curves that are disjoint from each other, and some intersection points, we can build a new Jordan curve which will bound the internal region.)

Proposition 5.1. Let $K_{1}, K_{2}, K_{3}, K_{4}$ be four pairwise touching Jordan regions whose pairwise intersections are non-empty arcs (which may be degenerate, that is a single point). Suppose that $K_{1} \cap K_{2} \cap K_{3} \cap K_{4}=\emptyset$. Then one of them lies in the internal region surrounded by the other three.

Proof. We will call the image of the non-negative reals under an injective mapping into the plane an unbounded path if it is an unbounded subset of the plane. The image of 0 is the starting point of the unbounded path.

Assume that $K_{1}$ is not in the internal region surrounded by $K_{2}, K_{3}, K_{4}$. (Note that this is also the case when $K_{1} \cap K_{2} \cap K_{3} \neq \emptyset$, because then the internal region is empty.) Then there is a point $p_{1}$ on the boundary of $K_{1}$ that does not belong to either of the other three sets, and from which there is an unbounded path, $\gamma_{1}$ disjoint from the other three sets. Similarly, if $K_{2}$ is not in the internal region surrounded by the other three, then there is a point $p_{2}$ on the boundary of $K_{2}$ that does not belong to either of the other three sets, and from which there is an unbounded path, $\gamma_{2}$ disjoint from the other three sets. And the same holds for $K_{3}$ yielding $p_{3}$ and $\gamma_{3}$.

We may assume that $\gamma_{1}, \gamma_{2}$ and $\gamma_{3}$ are pairwise disjoint. Now, $\gamma_{1} \cup \gamma_{2} \cup \gamma_{3}$ partition the external region of $K_{1}, K_{2}, K_{3}$ into three parts. And $K_{4}$ is in one of these three parts. However, each part only intersects two of the sets $K_{1}, K_{2}, K_{3}$, which is a contradiction.

Remark 5.2. Although Proposition 5.1 has been observed in [3], their proof does not work for the special case when three of the bodies share a common point. Therefore we found it necessary to give a general proof.

Observe that the conclusion of Proposition 3.2 follows from Proposition 5.1. Indeed, we may assume that $K_{4}$ is in the internal region $I$ surrounded by $K_{1}, K_{2}, K_{3}$. Suppose that $n \geq 5$. Since $K_{5}$ touches $K_{4}$, it must also lie in $I$. On the other hand, $K_{5}$ touches $K_{1}, K_{2}, K_{3}$ at points that do not belong to $K_{4}$. Now, (bd $I) \backslash K_{4}$ is the union of three open arcs, and $K_{4}$ must have a point on at least two of these arcs to touch $K_{1}, K_{2}, K_{3}$. However, then the interior of $K_{5}$ intersects the interior of at least one set from $K_{1}, \ldots, K_{4}$, a contradiction. 


\section{Acknowledgements}

Open access funding provided by Eötvös Loránd University (ELTE). This work was made under the supervision of Márton Naszódi, who called my attention to this topic. I would like to express my gratitude for his aid, the corrections and all the useful discussions. I thank Géza Tóth the idea of the much shorter proof of Proposition 3.2. This research was supported by the ÚNKP-17-3 New National Excellence Program of the Ministry of Human Capacities.

Open Access. This article is licensed under a Creative Commons Attribution 4.0 International License, which permits use, sharing, adaptation, distribution and reproduction in any medium or format, as long as you give appropriate credit to the original author(s) and the source, provide a link to the Creative Commons licence, and indicate if changes were made. The images or other third party material in this article are included in the article's Creative Commons licence, unless indicated otherwise in a credit line to the material. If material is not included in the article's Creative Commons licence and your intended use is not permitted by statutory regulation or exceeds the permitted use, you will need to obtain permission directly from the copyright holder. To view a copy of this licence, visit http://creativecommons. org/licenses/by/4.0/.

Publisher's Note Springer Nature remains neutral with regard to jurisdictional claims in published maps and institutional affiliations.

\section{References}

[1] Pach, M.J., Swanepoel, K.: Arrangements of homothets of a convex body. Mathematika 63(2), 696-710 (2017)

[2] Arias-de Reyna, J., Ball, K., Villa, R.: Concentration of the distance in finite dimensional normed spaces. Mathematika 45, 245-252 (1998)

[3] Bezdek, A., Kuperberg, K., Kuperberg, W.: Mutually contiguous translates of a plane disk. Duke Math. J. 78(1), 19-31 (1995)

[4] Bezdek, K., Brass, P.: On k+ -neighbour packings and one-sided Hadwiger configurations. Beiträge Algebra Geom. 44(2), 493-498 (2003)

[5] Bezdek, K., Connelly, R.: Intersection points. Ann. Univ. Sci. Budapest. Eötvös Sect. Math. 31(1988), 115-127 (1989)

[6] Böröczky, K., Szabó, L.: Minkowski arrangements of circles in the plane. Rendiconti del Circolo Matematico di Palermo 70, 87-92 (2002)

[7] Bourgain, J., Milman, V., Wolfson, H.: On type of metric spaces. Trans. Am. Math. Soc. 294(1), 295-295 (1986)

[8] Böröczky, K., Szabó, L.: Minkowski arrangements of spheres. Monatshefte für Math. 141(1), 11-19 (2004)

[9] Danzer, L., Grünbaum, B.: Über zwei Probleme bezüglich konvexer Körper von P. Erdös und von V. L. Klee. Math. Z 79, 95-95 (1962)

[10] Fejes Tóth, L.: Minkowskian distribution of discs. Proc. Am. Math. Soc. 16(5), 999-1004 (1965) 
[11] Fejes Tóth, L.: Packings and coverings in the plane, Proc. Coll. Convexity (Copenhagen: KØbenhavns Univ). Mat. Inst. 1967, 78-87 (1965)

[12] Füredi, Z., Lagarias, J.C., Morgan, F.: Singularities of minimal surfaces and networks and related extremal problems in Minkowski space. Discrete Comput. Geom. 6, 95-106 (1990)

[13] Groemer, H.: Abschätzungen für die Anzahl der konvexen Körper, die einen konvexen Körper berühren. Monatshefte für Math. 65, 74-81 (1961)

[14] Lángi, Zs, Naszódi, M.: On the Bezdek-Pach conjecture for centrally symmetric convex bodies. Canad. Math. Bull. 52(3), 407-415 (2009)

[15] Naszódi, M.: On a conjecture of Károly Bezdek and János Pach. Period. Math. Hungar. 53(1-2), 227-230 (2006)

[16] Naszódi, M., Swanepoel, K.: Arrangements of homothets of a convex body II. Contrib. Discrete Math. 13, 116-123 (2017)

[17] Pach, J., Agarwal, K.P.: Combinatorial Geometry. Tech. Report, Durham, NC, USA (1991)

[18] Petty, C.M.: Equilateral sets in Minkowski spaces. Proc. Am. Math. Soc. 29, 369-374 (1971)

[19] Polyanskii, A.: Pairwise intersecting homothets of a convex body. Discrete Math. 340(8), 1950-1956 (2017)

[20] Schneider, R.: Convex bodies: the Brunn-Minkowski theory, expanded ed., Encyclopedia of Mathematics and its Applications, vol. 151, Cambridge University Press, Cambridge, (2014)

[21] Thompson, A.C.: Minkowski Geometry. Encyclopedia of Mathematics and its Applications. Cambridge University Press, Cambridge (1996)

Viktória Földvári

ELTE Eötvös Loránd University

Budapest

Hungary

e-mail: foldvari@math.elte.hu

Received: December 3, 2019.

Revised: April 2, 2020. 